\title{
Dynamic modelling and torque ripple minimization of a lightweight ultra-high transmission ratio harmonic drive
}

\author{
Efstratios Tsolakis ${ }^{1, *}$, Georgios Vasileiou ${ }^{1}$, Nikolaos Rogkas $^{1}$, Christos Kalligeros ${ }^{1}$, Pavlos Zalimidis ${ }^{2}$ and Vasilios Spitas ${ }^{1}$ \\ ${ }^{1}$ Machine Design Laboratory, School of Mechanical Engineering,National Technical University of Athens,9 Heroon Polytechniou Str., \\ 15780 Zografou, Greece \\ ${ }^{2}$ School of Pedagogical and Technological Education Department of Mechanical Engineering Educators Iraklio Attikis, 14121 Athens, \\ Greece
}

\begin{abstract}
Harmonic drives have been of significant importance in many industrial and high-end applications including robotics, aerospace and manufacturing. Their unique characteristics combine high torque capabilities, high to ultra-high transmission per stage and low backlash performance in compact designs, suited for limited space applications. However, apart from the eminent merits of the technology, this type of gearboxes is associated with complex dynamic performance. Since their operations is associated with high compliance and friction a non - linear behavior is imposed to the system. This is also intensified due to torque ripples which in many cases apart from adding up to the overall complexity of the system, can also interfere with components of similar resonance frequency. In the frame of this paper a novel concept for a low-cost lightweight plastic harmonic drive used in positioning of telecommunication antennas is presented. Due to the tight dynamic specifications of the application (settling time, positioning error etc.) the torque ripple of the transmission was modelled and minimized. Design changes were also incorporated in various features in order to improve the overall dynamic performance.
\end{abstract}

\section{Introduction}

Harmonic drives have been of significant importance in many industrial and high-end applications including robotics, aerospace and manufacturing since their introduction in 1950s [1]. They combine high torque capabilities, high to ultra-high transmission ratio and near-zero backlash performance in compact designs, suited for limited space applications [2-5].

The unique characteristics of harmonic drives enable their effective use in applications that require high motion accuracy and speed reduction. In this paper a novel power transmission is designed based on the harmonic drive technology to meet the requirements of a pioneering system for the rotation and angular positioning of mobile phone antennas. The system consists of two identical speed reduction stages with the same transmission ratio. At each stage a pair of ring gears with non-standard gear teeth is responsible for the power transmission. The design material of the ring gears was chosen to be the high-performance polyphenylene sulfide thermoplastic (PPS).

The two main features of the reducer, namely the large transmission ratio and zero backlash, are achieved through the nature of harmonic drive technology, the special kinematics of which allow the power transfer to be distributed to a large number of gear teeth, per instantaneous rotation pole. For the proposed design, the number of teeth simultaneously involved in power transmission is equal to $14-$ a number unusually large for most applications of power transmission systems. The resulting load distribution leads to the ability to high output torque values transfer without failure. In addition, at any given time, at least two pairs of the teeth involved are in contact on both flanks without blocking their movement, thanks again to the harmonic drive kinematics, thus achieving zero backlash.

The ultra-high transmission ratio and zero backlash were the basic requirements of the proposed transmission because of the high levels of accuracy that the rotation and angular displacement on which the mobile phone antennas operate. More specifically, zero backlash responds to the requirement for robustness in antenna support, since even a deviation of a few seconds of a degree of the antenna results into extremely large deviations in the signal emitted. The large reduction in speed is due to the limited power available, due to the nature of the power electronics in these applications, which exclude high power limits in order to avoid inducing noise to the transmitted signal. 
The flexibility of the transmission and the kinematic principle of harmonic drives are responsible for the aforementioned unique characteristics which are necessary or even vital for many applications. However they are, also, responsible for some shortcomings in their operation. The transmission flexibility and its sensitivity to assembly errors such as misalignments cause torque ripples in the output of the harmonic drive and the increased friction forces can reduce the output accuracy of the system. The minimization of the torque ripples in harmonic drives has been the subject of thorough investigation by the scientific community [6-10]. Various control schemes have been developed and tested to compensate the ripples in the output torque. Classic controllers, such as PID controllers, are sensitive to parameter tuning and fail to apply effective control in several applications. On the contrary, modern types of control that are based to full-state feedback or the adaptive control seem to offer a more robust control solution.

In this paper a full-state feedback control approach has been adopted. By using a torque sensor and an observer to estimate in real time the state of the closed loop system a control scheme is designed that can minimize effectively the torque ripples, as presented in Sector 6.

\section{Benchmarking}

The final choice of Harmonic Drive technology was made after examining the state-of-the-art technology in the field of mechanical power transmission and speed reduction. Through this process, it was concluded that although there are a number of reliable and high-level solutions that can meet each of the above characteristics, difficulties occur when they should be address simultaneously. The reducers that are currently used in similar applications are mainly the following:

Classic multi-stage gearbox: Such reducers are characterized by a large number of components (it would take about 5-6 steps of gear stages to achieve the desired transmission ratio) and therefore their size and weight are prohibitively large. In addition, it is practically impossible to achieve zero backlash and even its minimization implies increased processing costs and therefore they are considered unsuitable for large production.

Epicyclic gearing: These are solid speed reducers, capable of achieving a large transmission ratio and transporting high load values, but even in this category, their operation is associated with backlash. If it is desired to be minimized, then the cost of construction and assembly is extremely high, which in turn is unsuitable for large production.

Cyclo-Drives: Reductors of this type are not based on the use of teeth but on a highly intelligent kinematics that leads to the transfer of power through many involved parties. However, this category has the same disadvantages as the above, in terms of high production and processing costs.

The advantages of the final solution of Harmonic Drive were already mentioned from the introduction and as can be seen from the above, they were enough to lead to his election.

\section{Technical requirements}

The technical requirements of the system for the rotation and angular displacement of mobile phone antennas were the following: Its rotation from end to end corresponds to an angle of $120^{\circ}$. The full rotation time must be $3 \mathrm{~min}$. The wind speed that the system is called to withstand is $50 \mathrm{~m} / \mathrm{s}$ based on EuroCode5, which translates to an existing torque of $1700 \mathrm{Nm}$. Since the entire system will be attachable, the overall size and weight of the harmonic drive will need to be low. Reverse rotation of the harmonic drive is prohibited. The combination of the above elements led to the design of a harmonic drive that would achieve a speed reduction of $3000 / 1$. In addition, due to the competitive values that the system is called to have, there was a need that the cost of construction per unit should remain low and always relevant to the total cost of the entire mechanism. Therefore, there is a need to reduce the components of the harmonic drive, which would require high tolerances and/or increased processing times, to a minimum.

Taking this considerations into account a novel fully plastic harmonic drive was designed following the layouts depicted below.

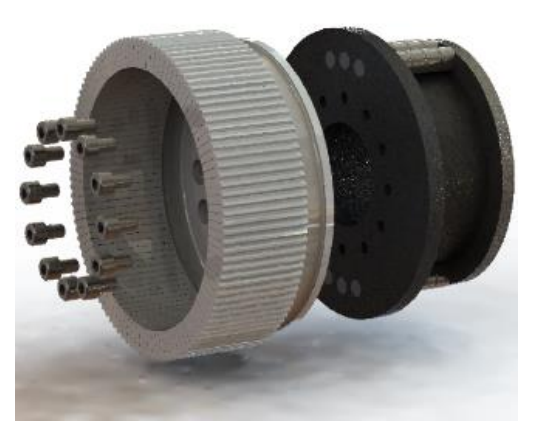

Fig. 1. Harmonic drive stage (flex spline \& wave generator). 


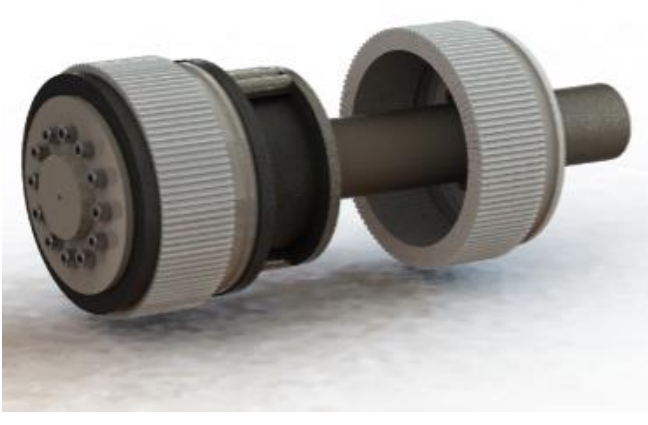

Fig. 2. Harmonic drive two stage assy.

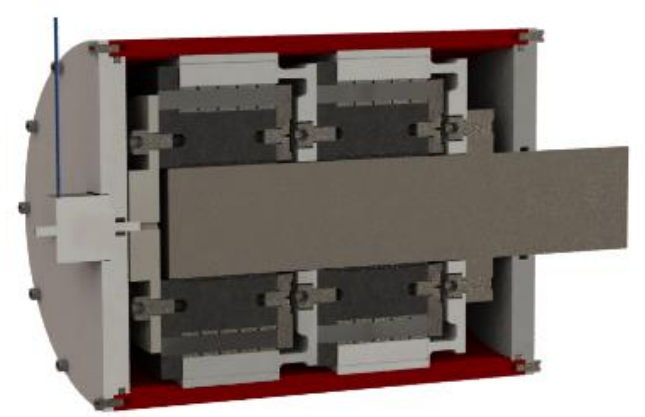

Fig. 3. Harmonic drive complete assembly.

The following chapters would present in detail the key features of that mechanism and would also describe the way the key challenge of the torque ripple was tackled.

\section{Kinematic analysis}

The specifications show that the speed of rotation of the antenna and therefore the output of the reducer is:

$$
\omega_{\text {out }}=\frac{\frac{2 \pi}{3} \mathrm{rad}}{3 \mathrm{~min}}=0.0166 \frac{\mathrm{rad}}{\mathrm{s}}
$$

In addition, for the output torque:

$$
M_{\text {out }}=1700 \mathrm{Nm}
$$

For the reducer designed the pair of flex spline-gear rings, they are shown in the figure below.

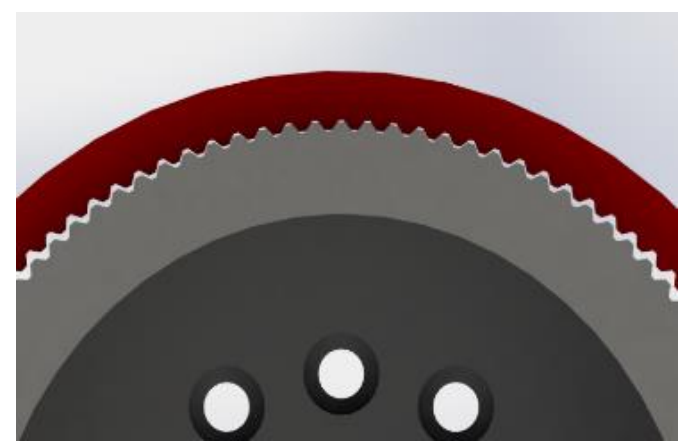

Fig. 4. Multiple teeth engagement.
The values of the number of teeth were selected and therefore the transmission ratios were as follows:

$$
\begin{gathered}
Z_{\text {out }}=110 \\
Z_{\text {out }}=108 \\
i=\frac{Z_{\text {out }}}{Z_{\text {out }}-Z_{\text {in }}}=\frac{110}{110-108}=55 \\
i_{01}=i_{02}=i \\
i_{\text {tot }}=i_{01} i_{12}=55 \times 55=3025
\end{gathered}
$$

Now calculating the power conservation for each stage we obtain the following relationships:

$$
\begin{gathered}
P_{\text {stepper }}=P_{\text {in }}=M_{\text {in }} \omega_{\text {in }} \\
P_{01}=M_{01} \omega_{01} \\
P_{12}=M_{12} \omega_{12}
\end{gathered}
$$

Where:

- $\quad \omega_{\text {in }}=$ engine speed

- $\omega_{01}=$ the speed of rotation at the output of the first flex spline

- $\omega_{12}=$ the rotation speed at the output of the second flex spline

For the completeness of the balance sheet, of course, the losses at each level are taken into account, which are practically perceived through the individual performance ratios. Thus, we have

- $\mathrm{n}_{01}=$ the efficiency of the first stage

- $\mathrm{n}_{12}=$ the efficiency of the second stage

Combining now the rotational speeds per stage as well as the transmission ratios we have:

$$
\begin{aligned}
& \omega_{01}=\frac{\omega_{\text {in }}}{i_{01}} \\
& \omega_{12}=\frac{\omega_{01}}{i_{12}}=\frac{1}{i_{12}} \frac{\omega_{\text {in }}}{i_{01}} \\
& \omega_{\text {out }}=\omega_{12}=\frac{\omega_{\text {in }}}{i_{\text {tot }}} \\
& \omega_{\text {in }}=\omega_{\text {out }} i_{\text {tot }}
\end{aligned}
$$

From the relationships that correlate the power of each stage with the corresponding efficiency we obtain:

$$
\begin{aligned}
& P_{\text {stepper }}=\frac{P_{12}^{\text {out }}}{n_{\text {tot }}} \\
& M_{\text {stepper }} \omega_{\text {stepper }}=\frac{M_{\text {out }} \omega_{\text {out }}}{n_{\text {tot }}}
\end{aligned}
$$

We therefore come to the two basic relationships that determine the characteristics of the engine required to meet the relevant specifications:

$$
\begin{gathered}
M_{\text {stepper }}=\frac{M_{\text {out }}}{n_{\text {tot }} i_{\text {tot }}}=\frac{1700}{0.75 \times 3025}=0.75 \mathrm{Nm} \\
\omega_{\text {stepper }}=\omega_{\text {out }} i_{\text {tot }}=5.58 \mathrm{RPS}
\end{gathered}
$$

Among the values already mentioned are: 
- $\mathrm{M}_{\text {out }}=1700 \mathrm{Nm}$

- $\mathrm{i}_{\mathrm{tot}}=3025$

Finally, regarding the total efficiency, a standard for plastic speed reducers (main loss resulting from the internal lag of the elastic parts) was used:

- $\quad$ ntot $=0.75$

Therefore, the motor needs to fulfil the following specificiations:

- $\quad \omega s t e p p e r=5.58 \mathrm{RPS}$

- $\quad$ Mstepper $=0.75 \mathrm{Nm}$

\section{Loading and stress analysis}

This chapter lists the critical parts of the final model, so that when transitioning from the design phase to the prototype phase issues regarding the strength of the various components are not neglected or underestimated. Therefore, endurance and loading for the following parts of the reducer were studied and calculated:

- Loading of flex spline at pre-tension phase

- Loading of flex spline during assembling

- $\quad$ Loading of bolts

- Wear of journal bearings

- Wear of roller bearings

The following segments concentrates on the Finite Element Analysis (FEA) carried for the aforementioned critical parts.

\subsection{Loading of flex spline at pre-tension phase} As already mentioned, the power transmission is achieved through the cooperation of a flexible inner and a rigid outer ring gear. The kinematics of the mechanisms require the inside ring gear (flex spline) to have two gear teeth less than the outside. This practically means that the length of its flex spline's pitch circle will be shorter by 2 circular pitches compared to the outside ring gear. In order to mesh the flex spline changes its shape from circular to elliptical thus growing in diameter and radius. This initial radial displacement is done through the wave generator, which adapts internally to the flex spline with a press fit, imposing its final shape. During this phase, the stress of flex spline is not negligible. Specifically, by imposing this pre-tension on the designed $16 \mathrm{~mm}$ flex spline, the material as shown in Figure 5 was led to failure, as the growing stresses exceeded the limit of $150 \mathrm{MPa}$, PPS has tensile strength.

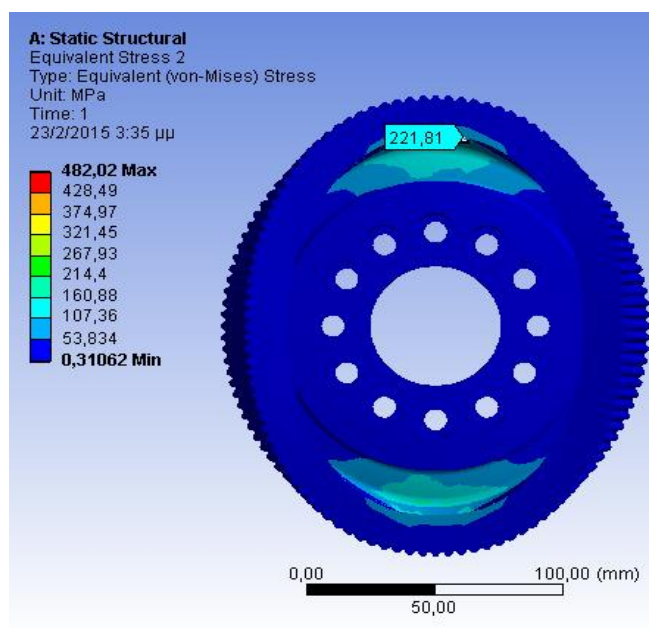

Fig. 5. Flex spline failure during pre-tension.

What was finally decided was to design some notches on the flex spline, as shown below, which, without affecting the ability to transmit torque to its main body, reduced the force required to impose the radial displacement, as they practically reduced the thickness of the active cross section participating in it.

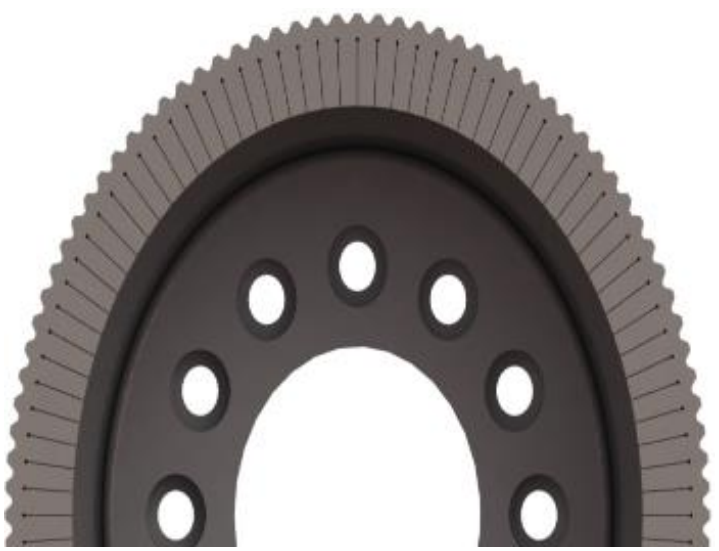

Fig. 6. Design modification (notches).

In addition, to reduce the stresses developed in the back of the flex spline, fillets were designed both internally and externally.

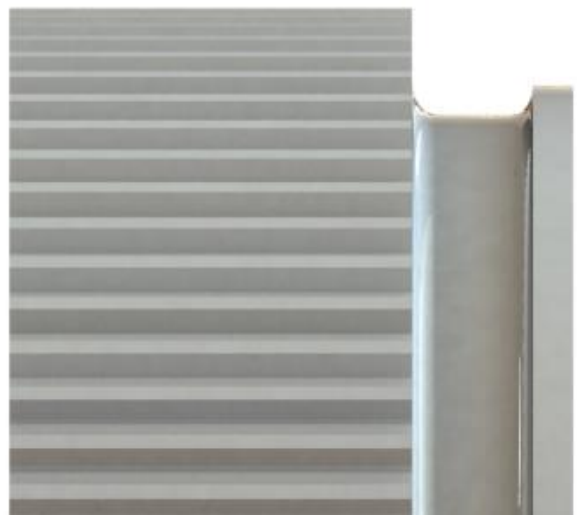

Fig. 7. Design modification back fillet 
The final values of the emerging stresses after the modifications are shown in the figures below.

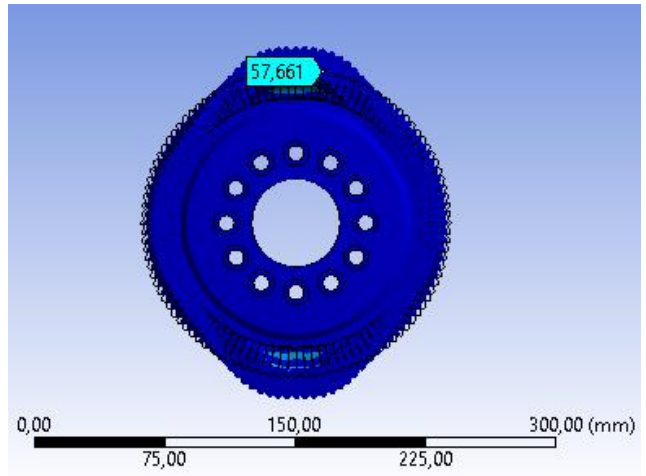

Fig. 8. Flex spline stressed condition.

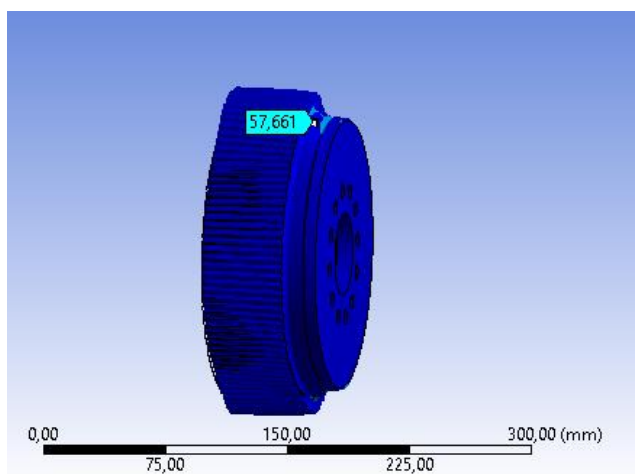

Fig. 9. Flex spline stressed condition (detail).

5.2 Flex spline deformation during engagament: If the flexible spline has the desired elliptical shape through radial prestressing, then it is able to transfer torque. Power is transmitted through the engaged involved which as was already mentioned the teeth involved in one side of the crown are 7, so a total of 14 .

Once the flexible spline is engaged, then of the strain, the teeth themselves will be strained in addition to the pre-stressed main body. This stress consists in both bending and surface pressure. In the following figures 10 and 11 it appears that the final stress of the flex spline does not exceed the limit value for the PPS, which, as mentioned, in attraction reaches $150 \mathrm{MPa}$.

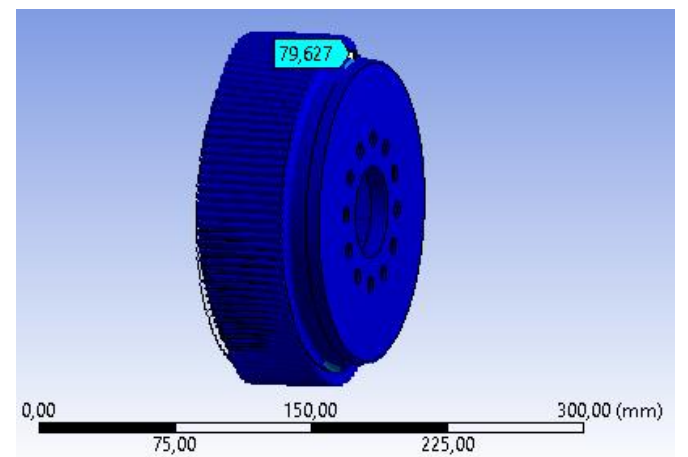

Fig. 10. Flex spline combined stressed condition.

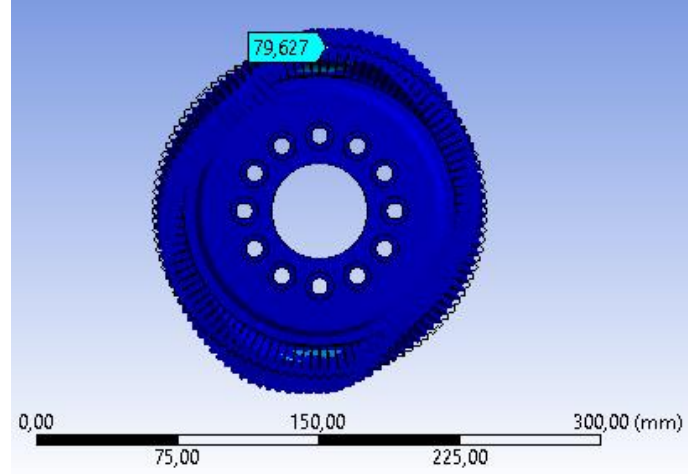

Fig. 11. Flex spline combined stressed condition (detail).

\section{Torque ripple minimization}

A significant shortcoming of harmonic drives is their sensitivity to geometrical deviations and assembly errors such as misalignments, which result to the occurrence of ripples in the output torque [6]. Given the lower stiffness of the proposed harmonic design due to the usage of thermoplastic materials for both ring gears, the amplitude of these torque ripples is expected to be higher than normal and potentially creating unstable operation and vibrations. Consequently, the minimization of their effect is of great significance for stable and accurate power transmission.

This Section presents the design of a control system that compensates for the ripples emerging in the output torque signal of a Harmonic Drive. The signal can be monitored in real time by integrating a torque sensor to the system. The control scheme should be able to minimize the errors between the measured signal and the reference signal. However, since the torque requirements and the dynamic characteristics of Harmonic Drives vary significantly for different applications, classic controllers such as PID fail to minimize successfully the torque ripples at all times. For this reason, modern types of control are being implemented, such as full-state feedback and adaptive control [7].

In order to design the control system, the dynamic characteristics of the system should be identified. Lu and Lin [6] obtained the transfer function of a Harmonic Drive power transmission by conducting testing on an existing experimental setup. The linearized transfer function of the system is presented in Eq. (19), where $y(s)$ is the Laplace transform for the harmonic drive's output torque, while $u(s)$ is the Laplace transform for the input (motor) torque. 


$$
\begin{aligned}
& G(s)=\frac{y(s)}{u(s)}= \\
& =\frac{4.83 e 10}{s^{4}+998.95 s^{3}+1.22 e 6 s^{2}+7.28 e 7 s+4.84 e 10}
\end{aligned}
$$

The non-linearities that the torque ripples introduce to the system are added as a distribution to the input signal, as shown in Fig. 12. A special characteristic of the torque ripples developed in harmonic drives is that their dominating component has a frequency twice larger than the angular speed of the input shaft [10]. Consequently, the disturbance signal caused by the torque ripples can be simulated as a sum of sinusoids (Eq. 20) with a frequency of $2 \omega_{i}$, where $\omega_{i}$ is the rotational speed of the input shaft. The coefficients $a_{j}$ and $b_{j}$ are generally unknown and dependent on the system and the application.

$$
d(t)=\sum_{j=1}^{n} a_{j} \cos \left(2 \omega_{i} t\right)+b_{j} \sin \left(2 \omega_{i} t\right)
$$

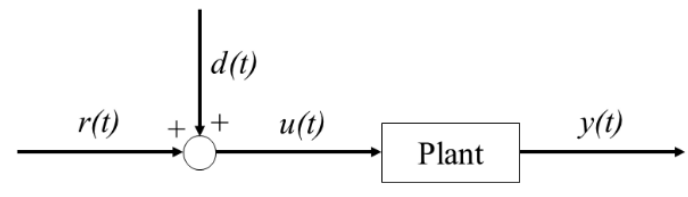

Fig. 12. Open-loop Harmonic Drive system modeling.

The proposed control system combines the full-state feedback control scheme with an additional free integrator. This design provides robustness to the control system since it is considerably less sensitive to uncertainties and disturbances. However, there are three prerequisites in order to implement this type of control to a plant; it has to be controllable and contain no zeros or poles that equal 0 . In this case, the plant, namely the Harmonic Drive, given its transfer function in Eq. (19), is a Linear Time Invariant (LTI) system thus controllable and 0 is neither one of its poles nor on one of its zeros.

The open-loop system contains 4 degrees of freedom (DOF) and its state-space equations are given in Eq. (21). The final closed-loop system has an additional DOF due to the integrator of the controller. The new state-space equations are given in Eq. (22). Since the final system has 5 DOF but only one is accessible through the torque sensor, a straightforward full-state feedback control is impossible. However, since every LTI system is also observable, an observer can be designed and implemented in order to estimate in real time the full-state of the closed loop. The final control scheme is presented in Fig. 13. The gains $K^{*}$ and $L$ for the controller and the observer respectively are calculated by Ackermann's formula presented in Eq. (23) and (24). The new closed-loop matrices $A^{*}$ and $B^{*}$ for the calculation of $K^{*}$ are given in Eq. (25), while $a(A)$ is the desired characteristic polynomial evaluated at matrix $A$.

$$
\begin{aligned}
& \left\{\begin{array}{c}
x \dot{(t)}=A x(t)+B u(t) \\
y(t)=C x(t)
\end{array}\right. \\
& {\left[\begin{array}{c}
\dot{x}(t) \\
\dot{\xi}(t)
\end{array}\right]=\left[\begin{array}{cc}
A-B K & B k_{1} \\
-C & 0
\end{array}\right]\left[\begin{array}{l}
x(t) \\
\xi(t)
\end{array}\right]+\left[\begin{array}{l}
0 \\
1
\end{array}\right] r(t)} \\
& y(t)=\left[\begin{array}{ll}
C & 0
\end{array}\right]\left[\begin{array}{l}
x(t) \\
\xi(t)
\end{array}\right] \\
& K^{*}=\left[\begin{array}{ll}
K & k_{1}
\end{array}\right] \\
& =\left[\begin{array}{lllll}
0 & 0 & 0 & 0 & 1
\end{array}\right] P^{-1}\left(A^{*}, B^{*}\right) a\left(A^{*}\right) \\
& L=a(A) Q^{-1}(A, B)\left[\begin{array}{lllll}
0 & 0 & 0 & 0 & 1
\end{array}\right]^{T} \\
& A^{*}=\left[\begin{array}{cc}
A & B \\
-C & 0
\end{array}\right], B^{*}=\left[\begin{array}{c}
B \\
0
\end{array}\right]
\end{aligned}
$$

Fig. 13. Closed-loop control system.

Two dominant poles are selected for the closed loop system, as presented in Eq. (26). The remaining poles are placed at least 10 times faster. The poles of the observer are selected 10 times faster than those of the system for the fast convergence of the state estimation.

$$
\lambda_{1,2}=-\zeta \omega_{n} \pm j \omega_{n} \sqrt{1-\zeta^{2}}
$$

where $\omega_{n}$ is the natural frequency and $\zeta$ the damping ratio. 
To demonstrate the capabilities of the control scheme, the closed-loop system of Fig. 13 has been constructed in numerical platform of MatLab/ Simulink and a sinusoidal input reference signal has been given. The input signal indicate the required torque at the output of the Harmonic Drive. As a result, the reference signal has the frequency of the input shaft divided by the transmission ratio of the stage which is set to $N=50$.

In Fig. 14 the open-loop and closed-loop responses of the system are presented and compared. For the poles of the closed loop a damping ratio of 0.69 and a natural frequency 10 times the input shaft frequency have been selected. It is obvious that the proposed control scheme can minimize the torque ripples and provide a very accurate tracking of the response signal.

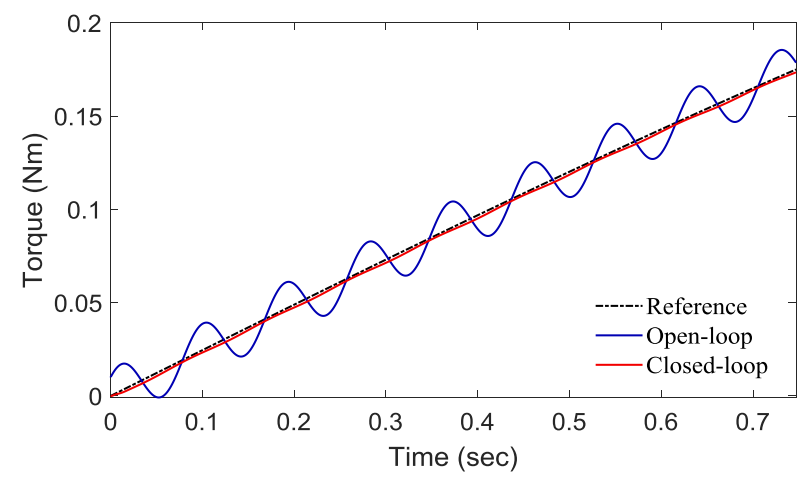

Fig. 14. Tracking response of the proposed control system in comparison with the open-loop response.

To achieve the accurate tracking of the reference signal in Fig. 14, high gains are required, increasing the cost and the sensitivity of the control system. In Fig. 15 and 16 the output of the system is presented for different pole placement. In Fig. 15 different natural frequencies of the poles have been tested in respect to the input shaft speed, while in Fig. 16 the poles of the observer have been altered to $10,5,2$ and 1 times faster than those of the system. The closer the poles are to the imaginary axis, the less minimization of the torque ripples is achieved, with the phenomenon more apparent with the poles of the closed-system. The final pole placement for the controller and the observer depends on the requirements of every application. Regarding this paper, a natural frequency 5 times bigger the input shaft angular velocity is selected for the closed-loop system poles, while the poles of the observer are chosen to be 2 times faster.

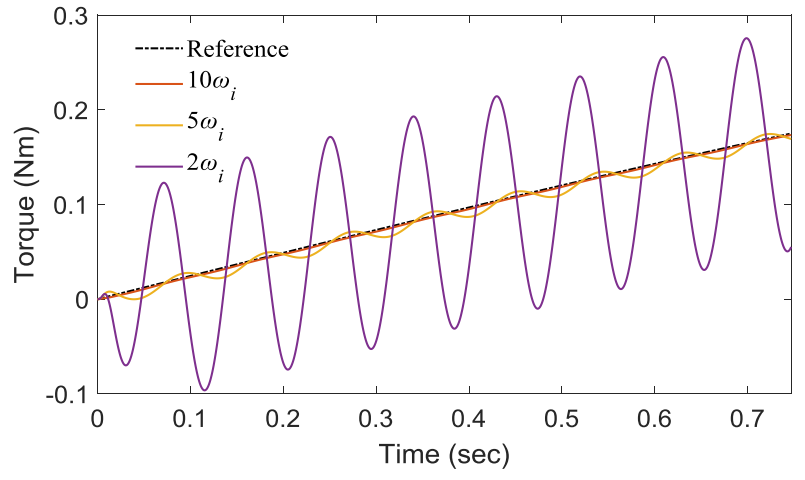

Fig. 15. Tracking response of the close-loop system according to different closed-loop poles.

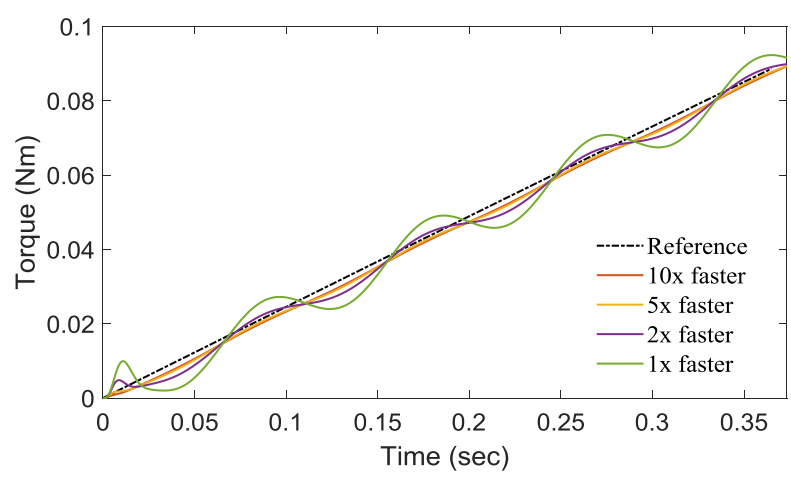

Fig. 16. Tracking response of the close-loop system according to different observer poles.

\section{Results and discussion}

Closing the paper summarizes the data of the designed reducer, which qualify it as the final solution for its application to the rotating mechanism. Final transmission ratio achieved $\mathrm{i}=$ 3025. Ability to transfer output torque $>1700 \mathrm{Nm}$. Total weight $<11 \mathrm{~kg}$. Minimum required volume (Ø200 X $250 \mathrm{~mm}$ ) Design based on cast-in-place and interchangeable components in order to minimize construction costs. Participation of at least 14 teeth per pole of rotation and therefore distribution of the load bearing.

Zero backlash due to permanent involvement of at least 2 teeth per pole of rotation. Non-reversible operation resulting from self-stability, thus eliminating the need for a braking mechanism. Easy adjustment of reducer to the rest of the construction. Minimization of output torque ripples through a control scheme based on the full-state feedback control. A torque sensor provide the measurement for the control system and an observer provide the real-time estimation of the state of the closed-loop system. 


\section{References}

1. C. W. Musser. (1955). "Strain wave gearing," U.S. Patent $2906 \quad 143 \quad 03$ 23, [Online]. Available:http://www.google.com/patents?vid= 2906143

2. Routh, B. (2018, April). Design aspects of harmonic drive gear and performance improvement of its by problems identification: A review. In AIP Conference Proceedings (Vol. 1943, No. 1, p. 020016). AIP Publishing LLC.

3. Buss, A., \& Trhon̆, V. (2014). Harmonic Drive products in robotic. In Applied Mechanics and Materials (Vol. 613, pp. 112-116). Trans Tech Publications Ltd.

4. Ueura, K., \& Slatter, R. (1999). Development of the harmonic drive gear for space applications. European Space AgencyPublications-ESA SP, 438, 259-264.

5. Dhaouadi, R., \& Ghorbel, F. H. (2008). Modelling and analysis of nonlinear stiffness, hysteresis and friction in harmonic drive gears. International Journal of Modelling and Simulation, 28(3), 329-336.

6. Lu, Y. S., \& Lin, S. M. (2007). Disturbanceobserver-based adaptive feedforward cancellation of torque ripples in harmonic drive systems.Electrical Engineering, 90(2), 95-106.

7. Lu, Y. S., Lin, S. M., Hauschild, M., \& Hirzinger, G. (2013). A torque-ripple compensation scheme for harmonic drive systems. Electrical Engineering, 95(4), 357365.

8. Taghirad, H. D., \& Belanger, P. R. (1998). Torque ripple and misalignment torque compensation for the built-in torque sensor of harmonic drive systems. IEEE Transactions on Instrumentation and Measurement, 47(1), 309315.

9. Godler, I., Ninomiya, T., \& Horiuchi, M. (2001). Ripple compensation for torque sensors built into harmonic drives. IEEE Transactions on Instrumentation and Measurement, 50(1), 117-122.

10. Hidaka T, Zhang Y, Sasahara M, Tanioka $Y$ (1989) Vibration of a strain wave gearing in an industrial robot. In: Proceedings of ASME International Power Transmission and Gearing Conference, pp 789-794 